\title{
Characterization of the immune responses elicited by baculovirus-based vector vaccines against influenza virus hemagglutinin
}

\author{
Zhi-peng HU, Juan YIN, Yuan-yuan ZHANG, Shu-ya JIA, Zuo-jia CHEN, Jiang ZHONG* \\ Department of Microbiology and Microbial Engineering, School of Life Sciences, Fudan University, Shanghai 200433, China
}

\begin{abstract}
Aim: To compare the specific immune responses elicited by different baculovirus vectors in immunized mice.
Methods: We constructed and characterized two recombinant baculoviruses carrying the expression cassette for the H5N1 influenza virus hemagglutinin (HA) gene driven by either an insect cell promoter (vAc-HA) or a dual-promoter active both in insect and mammalian cells (VAc-HA-DUAL). Virus without the HA gene (VAc-EGFP) was used as a control. These viruses were used to immunize mice subcutaneously and intraperitoneally. The production of total and specific antibodies was determined by ELISA and competitive ELISA. Cytokine production by the spleen cells of immunized mice was studied using the ELISPOT assay.

Results: Both the vAc-HA and vAc-HA-DUAL vectors expressed HA proteins in insect Sf9 cells, and HA antigen was present in progeny virions. The vAc-HA-DUAL vector also mediated HA expression in virus-transduced mammalian cell lines (BHK and A547). Both vAcHA and vAc-HA-DUAL exhibited higher transduction efficiencies than vAc-EGFP in mammalian cells, as shown by the expression of the reporter gene egfp. Additionally, both vAc-HA and VAc-HA-DUAL induced high levels of HA-specific antibody production in immunized mice; vAc-HA-DUAL was more efficient in inducing IFN- $y$ and IL-2 upon stimulation with specific antigen, whereas vAc-HA was more efficient in inducing IL-4 and IL-6.

Conclusion: Baculovirus vectors elicited efficient, specific immune responses in immunized mice. The vector displaying the HA antigen on the virion surface (VAc-HA) elicited a Th2-biased immune response, whereas the vector displaying HA and mediating HA expression in the cell (vAc-HA-DUAL) elicited a Th1-biased immune response.
\end{abstract}

Keywords: baculovirus; H5N1 avian influenza; hemagglutinin; immune response; vector vaccine

Acta Pharmacologica Sinica (2012) 33: 783-790; doi: 10.1038/aps.2012.23; published online 7 May 2012

\section{Introduction}

The H5N1 avian influenza virus is a highly contagious virus that causes significant morbidity and mortality worldwide. It infects not only avian species but also humans. Over 565 human cases have been reported, of which 331 cases were fatal $^{[1]}$. New prophylactic and therapeutic strategies are essential for pandemic influenza preparedness ${ }^{[2]}$.

The baculovirus Autographa californica multiple nucleopolyhedrovirus (AcMNPV) has been widely used to overexpress recombinant proteins in insect cells. Recently, it has also been found to enter mammalian cells efficiently and without viral replication. Modified AcMNPV can express exogenous genes in mammalian cells when controlled by promoters active in mammalian cells. The list of mammalian cells permissive to baculovirus transduction has expanded rapidly ${ }^{[3]}$. Because

\footnotetext{
* To whom correspondence should be addressed.

E-mail jzhong@fudan.edu.cn

Received 2011-12-05 Accepted 2012-02-29
}

of its excellent biosafety and high efficiency in gene delivery, baculovirus is believed to have great potential as a novel vector for gene therapy and vaccine development ${ }^{[3,4]}$.

Two basic approaches have been explored to develop baculovirus as a vaccine vector. One approach is to insert the expression cassette of the target antigen into the viral genome so that the recombinant virus can produce the antigen inside the host cells. The second approach is to display the antigen on the virion surface. Both approaches have been shown to elicit efficient immune responses against target antigens in vivo ${ }^{[5-8]}$.

In the current study, two recombinant baculoviruses were constructed, one displaying the influenza virus HA protein only on the virion surface, and the other both displaying HA and mediating HA expression in mammalian cells. Although both viruses elicited efficient responses in immunized mice, they showed different biases with regard to Th1 and Th2 balance. Our results provide new insight into the immune responses elicited by baculovirus vectors and indicate that 
baculoviruses can be used as efficient vectors for vaccine development ${ }^{[9,10]}$.

\section{Materials and methods}

\section{Construction of recombinant baculovirus vectors}

The pFB-EGFP plasmid was constructed as follows. The enhanced green fluorescence protein gene (egfp), driven by the tandemly arranged promoters of baculovirus p10 and CMV IE1, was digested from $\mathrm{pFB}-\mathrm{GR}$ using $\mathrm{X} b a \mathrm{I}$ and inserted into the $\mathrm{X} b a$ I site of pFastBac1 (Invitrogen, Carlsbad, CA, USA). The full-length HA gene from the H5N1 highly pathogenic avian influenza virus was constructed from the HA gene sequence of A/Vietnam/1203/2004 (GenBank accession № AY818135) by annealing synthetic oligonucleotides and amplifying with PCR. The full-length gene was verified by DNA sequencing; it was then inserted into pFB-EGFP between the $\mathrm{BamH} \mathrm{I}$ and Sal I sites downstream of the baculovirus polyhedrin promoter to generate the plasmid pFB-HA.

The pFB-HA-DUAL plasmid was constructed by inserting the CMV IE1 promoter into the BamH I site between the polyhedrin promoter and the HA coding sequence in $\mathrm{pFB}$ HA. The CMV IE1 promoter was generated by PCR using pFB-EGFP as the template and the primers $5^{\prime}$-GGCGGATCCCGTTACATAACTTACGGTAAATGGCCCGCCTGGC-3' and $5^{\prime}$-GCCGGATCCCGGTGTCTTCTATGGAGGTCAAAACAGCGTGG-3'.

\section{Virus and cells}

The recombinant baculoviruses, vAc-EGFP, vAc-HA, and vAc-HA-DUAL, were generated from the plasmids, $\mathrm{pFB}-$ EGFP, pFB-HA, and pFB-HA-DUAL, respectively, using the Bac-to-Bac system (Invitrogen) according to the manufacturer's instructions. Viruses were grown in Spodoptera frugiperda (Sf9) cells at a Multiplicity of Infection (MOI) of 0.1 (plaque forming units/cell) and harvested $4 \mathrm{~d}$ post-infection. Virus titers were determined by end-point dilution assay in Sf9 cells $^{[11]}$. Viruses in the supernatant were concentrated by centrifugation at $40000 \times g$ for $1 \mathrm{~h}$; pellets were suspended in PBS and further purified by $25 \%-60 \%$ sucrose gradient ultracentrifugation at $100000 \times \mathrm{g}$ for $1 \mathrm{~h}$. To determine the distribution of HA proteins, purified virions were treated with an equal volume of $1 \%$ Triton X-100 for 15 min to disrupt the viral envelope, and the viral nucleocapsids were collected by centrifugation at $50000 \times g$ for $1 \mathrm{~h}$.

Sf9 cells were cultured at $27^{\circ} \mathrm{C}$ in TNM-FH medium (SigmaAldrich, St Louis, MO, USA) supplemented with $10 \%$ fetal bovine serum (FBS), $100 \mu \mathrm{g} / \mathrm{mL}$ streptomycin and $100 \mathrm{U} / \mathrm{mL}$ penicillin. Baby hamster kidney (BHK) and human lung (A549) cell lines were cultured in Dulbecco's modified Eagle's medium (DMEM, Invitrogen) supplemented with $10 \% \mathrm{FBS}$, $100 \mu \mathrm{g} / \mathrm{mL}$ streptomycin, and $100 \mathrm{U} / \mathrm{mL}$ penicillin at $37^{\circ} \mathrm{C}$ and $5 \% \mathrm{CO}_{2}$.

\section{Baculovirus transduction of mammalian cells}

BHK or A549 cells were seeded in 24-well plates and cultured until the cells reached approximately $70 \%-80 \%$ confluence.
Then, the culture medium was removed, and the cells were washed three times with PBS (pH 7.4). The baculovirus inoculum was added to the cells to an MOI of 200, and the cells were incubated for $2 \mathrm{~h}$ at $37^{\circ} \mathrm{C}$. Virus was removed, fresh medium was added, and the cells were incubated at $37^{\circ} \mathrm{C}$ for another $24 \mathrm{~h}$ before the expression of HA was examined ${ }^{[12-14]}$.

\section{Western blot analysis}

Total protein from cell or virus samples was separated using $10 \%$ sodium dodecyl sulfate-polyacrylamide gel electrophoresis (SDS-PAGE) and transferred to polyvinylidene difluoride (PVDF) membranes. Mouse antibody against HA (H5-specific, 1:5000 dilution, USBiological, Swampscott, MA, USA) and alkaline phosphatase-conjugated goat anti-mouse IgG (1:30000 dilution, Sigma-Aldrich) were used as the primary and secondary antibodies, respectively. Blots were developed with NBT and BCIP.

\section{Flow cytometry}

BHK or A549 cells were transduced with baculovirus at an MOI of 10 for BHK cells and an MOI of 100 for A549 cells as described above, then cultured for $24 \mathrm{~h}$. The cells were detached by trypsinization, washed twice with PBS and analyzed for green fluorescence by flow cytometry (Becton Dickinson FACS Calibur). A minimum of 10000 events were collected and analyzed for each sample. Data were analyzed using the CELLQUEST software. Representative results from at least three independent experiments are shown.

\section{Immunization of mice}

Six- to eight-week-old female BALB/c mice (Animal Center, Shanghai Institutes for Biological Science, Shanghai, China) were randomly divided into three groups with 8 mice per group $(n=8)$. The mice were immunized twice, separated by $14 \mathrm{~d}$, both subcutaneously and intraperitoneally, with $1 \times 10^{9}$ pfu of recombinant baculovirus per inoculum. Immunized mice were housed in the SPF animal facility at Fudan University (Shanghai, China) under constant conditions of $23 \pm 1^{\circ} \mathrm{C}$, $40 \% \pm 5 \%$ humidity and a $12 \mathrm{~h}: 12 \mathrm{~h}$ light/dark cycle, with free access to pellet food and tap water. All animal experiments were performed in accordance with the Guide for the Care and Use of Laboratory Animals and were approved by the Ethics Committee for Animal Care and Use of Fudan University. One week following the second immunization, serum samples were collected from the retro-orbital plexus for serological testing and antibody assay; the mice were sacrificed, and splenocytes were harvested for ELISPOT assay.

\section{Enzyme-linked immunosorbent assay (ELISA) and competitive ELISA}

The expressed amount of HA-specific antibody in immunized mice was determined by competitive ELISA using an H5-subtype HA detection kit (AXIOM, Burstadt, Germany) and the negative and positive control samples provided by the kit. The levels of total IgG and IgA in the mice were also determined using ELISA kits (ADI, San Antonio, TX, USA). 
All steps were performed according to the manufacturer's instructions. The antibody concentrations in the samples were calculated from standard curves.

Splenocytes were harvested from the mice one week after the second immunization and cultured in 24-well plates $\left(1 \times 10^{6} /\right.$ well $)$ at $37^{\circ} \mathrm{C}$ and $5 \% \mathrm{CO}_{2}$ in the presence of one of the following: an HA-specific peptide (a 14-mer peptide from the middle region of $\mathrm{HA}, 5 \mu \mathrm{g} / \mathrm{mL}$, Biodesign, catalog number R88640), a non-specific peptide (a 16-mer peptide from the middle region of neuraminidase, $5 \mu \mathrm{g} / \mathrm{mL}$, Biodesign, catalog number R88440) or plant hemagglutinin (PHA, $2.5 \mu \mathrm{g} / \mathrm{mL}$ ). A control of untreated splenocytes was also included. After $72 \mathrm{~h}$ of incubation, the culture supernatant was harvested, and the level of IFN-Y and IL-4 in the supernatant was determined using mouse IFN- $\gamma$ and IL-4 immunoassay ELISA kits (Dakewei, Shenzhen, China) according to the manufacturer's instructions. The concentrations of IFN- $\gamma$ and IL-4 in the samples were determined using standard curves.

\section{ELISPOT assay}

The number of splenocytes producing cytokines upon antigen stimulation was determined using the ELISPOT assay (U-CyTech, Netherlands) according to the manufacturer's instructions. Briefly, 96-well plates were coated overnight at $4^{\circ} \mathrm{C}$ with $100 \mu \mathrm{L}$ per well of specific antibody against the cytokines of interest (IFN- $\gamma$, IL-2, IL-4, or IL-6). The plates were washed three times with sterile and pyrogen-free PBS and blocked with blocking buffer for $1 \mathrm{~h}$ at $37^{\circ} \mathrm{C}$. The splenocytes from immunized mice were added to the wells $\left(1 \times 10^{5} /\right.$ well) in RPMI-1640 containing either the HA peptide, the NA peptide or PHA as described above. Mock-stimulated and blank controls were included. The cells were cultured for $24 \mathrm{~h}$ at $37^{\circ} \mathrm{C}, 5 \% \mathrm{CO}_{2}$ and $100 \%$ humidity. Cultured cells were washed twice with PBS and 5 times with wash buffer (PBS with $0.05 \%$ Tween-20). Then, $100 \mu \mathrm{L}$ of properly diluted (1:10) biotinylated detection antibodies was added to each well, and the plate was incubated for $1 \mathrm{~h}$ at $37^{\circ} \mathrm{C}$. After washing both sides of the PVDF membrane 5 times with wash buffer, $100 \mu \mathrm{L}$ of properly diluted (1:10) streptavidin-HRP solution was added to each well, and the plate was incubated for $1 \mathrm{~h}$ at $37^{\circ} \mathrm{C}$. The plate was washed as described above and developed for $25 \mathrm{~min}$ at room temperature in the dark with the peroxidase substrate AEC. The reaction was stopped by thoroughly rinsing both sides of the PVDF membrane with deionized water. The plates were air dried at room temperature, and the spots were counted using an immunospot image analyzer. The blank control consistently demonstrated fewer than 10 SFC (spot forming cells) per $10^{6}$ splenocytes.

\section{Statistical analysis}

Means from at least 3 replicates are shown along with error bars representing the standard deviations. Animal experiments were repeated at least twice for each treatment. Student's $t$-test was used to compare humoral or cellular immune responses between different groups, and $P$-values less than 0.05 were considered statistically significant .

\section{Results}

\section{Construction of recombinant viruses}

Recombinant baculoviruses for the expression of H5N1 influenza virus HA protein in insect cells (vAc-HA), or in both insect cells and mammalian cells (vAc-HA-DUAL), were constructed. Both viruses contained an egfp-gene expression cassette for both insect and mammalian cells. The control virus, vAc-EGFP, contained the egfp gene but not the HA gene. The genomic structure of the three recombinant viruses is shown in Figure 1.

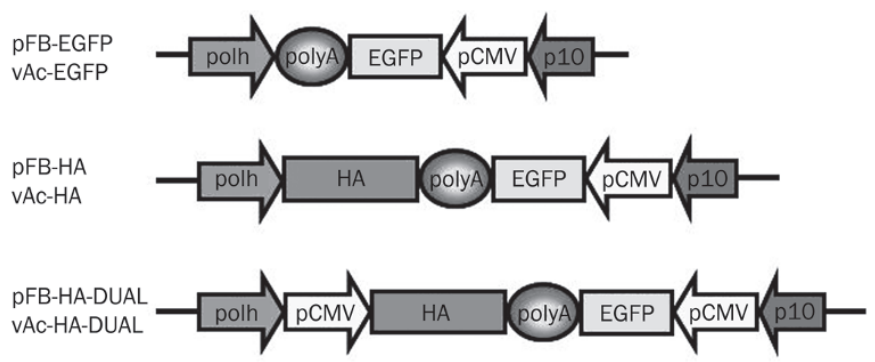

Figure 1. Schematic illustration of recombinant baculoviruses. The EGFP and $\mathrm{HA}$ expression cassettes were inserted in the polyhedrin locus.

\section{Presence of HA on the viral envelope}

Western blotting using anti-HA monoclonal antibodies revealed a protein of approximately $72 \mathrm{kDa}$ in Sf9 cells infected by vAc-HA and vAc-HA-DUAL (Figure 2A). By contrast, no HA protein was observed in cells infected by vAcEGFP. Mock-treated cells were uninfected.

Virions produced by the recombinant baculoviruses were purified from the medium of infected Sf9 cells and examined for the presence of HA protein using Western blotting. As shown in Figure 2B, HA proteins were found in vAc-HA and vAc-HA-DUAL virions but not in vAc-EGFP virions. When the virions were treated with Triton $\mathrm{X}-100$ to dissolve the viral envelopes, HA proteins were no longer present (Figure 2B), indicating that the HA proteins were localized to the viral envelope.

\section{Baculovirus-mediated HA expression in mammalian cells}

The recombinant baculoviruses vAc-EGFP, vAc-HA, and vAcHA-DUAL were used to transduce BHK cells at an MOI of 100. Green fluorescence was observed in cells transduced by all three viruses $24 \mathrm{~h}$ post-transduction (pt) (data not shown). As expected, Western blot analysis using anti-HA antibodies demonstrated HA expression only in BHK cells transduced with vAc-HA-DUAL (Figure 2C). The HA protein was partially cleaved to HA1 (55 kDa) and HA2 in BHK cells.

\section{Growth curves for recombinant baculoviruses}

The growth kinetics of recombinant baculoviruses in Sf9 cells were assayed, and the results are shown in Figure 3. Recombinant viruses expressing HA on their envelopes (vAc-HA and vAc-HA-DUAL) grew slower than the control virus (vAc- 
A
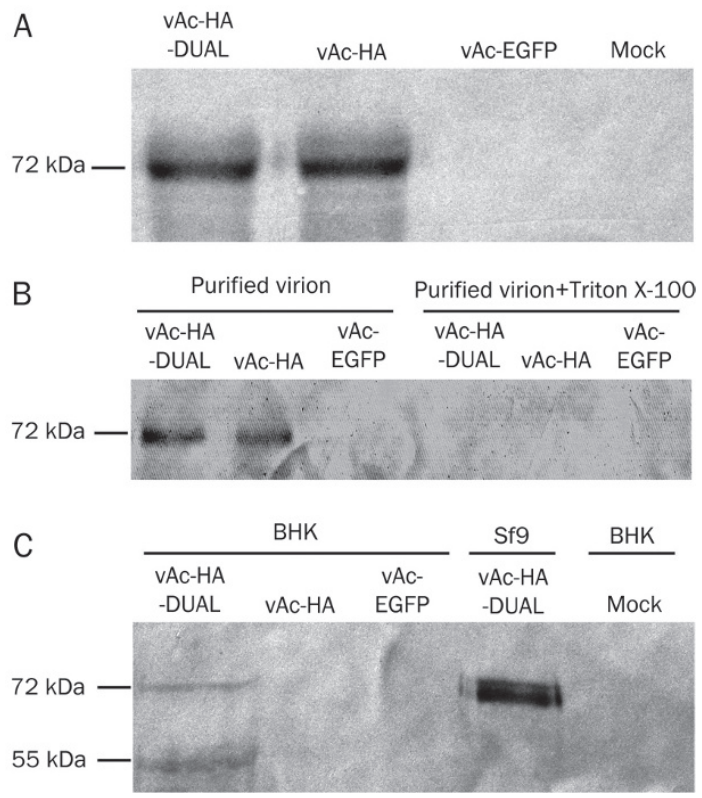

Figure 2. Expression of $\mathrm{HA}$ protein cells and its presence in the virion. (A) HA protein expressed in Sf9 cell. Total cellular extracts from Sf9 cells infected with VAc-HA-DUAL, VAc-HA, and VAc-EGFP were analyzed with SDS-PAGE and Western blot. Uninfected Sf9 cells were used as a mock control. (B) Localization of HA in the envelop of VAc-HA and VAc-HA-DUAL virus particles. Purified virion and virion treated with Triton X-100 to remove the envelop were examined for HA protein with Western blot. (C) Expression of the HA protein in baculovirus-transduced BHK cells. Sf9 cells infected with VAc-HA-DUAL and untransduced BHK cells were used as positive or negative controls, respectively. The HA protein expressed in BHK cells was partially cleaved to HA1 and HA2.

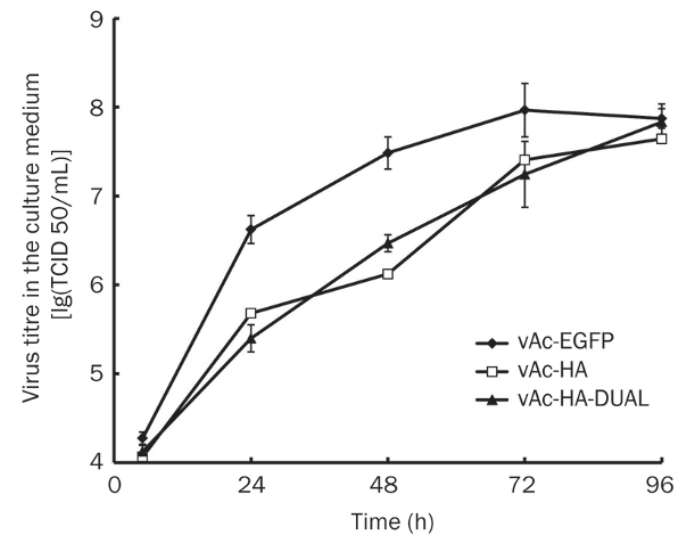

Figure 3. Virus growth curves in Sf9 cells. Sf9 cells were infected with vAc-EGFP, vAc-HA, and vAc-HA-DUAL at MOI 1. Each data point represents the mean value of 3 replicates.

EGFP), with a lag time of approximately $24 \mathrm{~h}$. However, the recombinants reached similar titers as vAc-EGFP late in the infection ( $96 \mathrm{~h}$ post-infection). Extensive syncytium formation was also observed in Sf9 cells infected with vAc-HA and vAcHA-DUAL (data not shown).

\section{Transduction efficiency of the HA-displaying baculoviruses}

BHK and A549 cells were transduced with recombinant baculoviruses at an MOI of 10 and 100, respectively. The cells were analyzed for green fluorescence using flow cytometry $36 \mathrm{~h} \mathrm{pt}$. As shown in Figure 4, the percentages of EGFP-positive cells in BHK cells transduced by vAc-HA and vAc-HA-DUAL were both twice as high as in cells transduced by vAc-EGFP. Similar differences were observed between HA-displaying recombinant viruses and the control virus in A549 cells. In both cell lines, similar percentages of EGFP-positive cells were observed with vAc-HA and vAc-HA-DUAL. These results indicate that the HA protein on the surface of recombinant baculoviruses preserved its native activity and contributed to viral entry into mammalian cells.

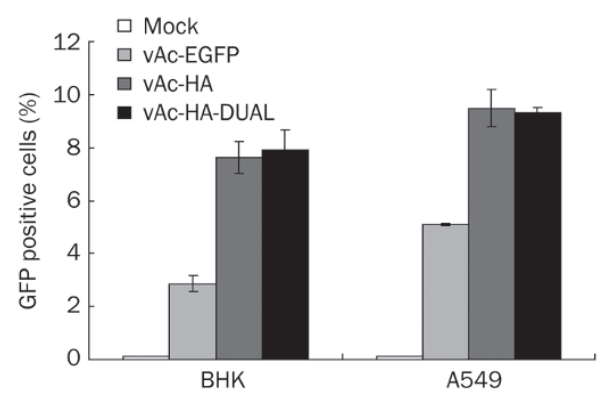

Figure 4. Transduction efficiency of HA-displaying baculoviruses. BHK and $A 549$ cells were transduced with baculoviruses at $\mathrm{MOI}$ of 10 and 100 , respectively, collected at $24 \mathrm{~h}$ post transduction, and analyzed by flow cytometry for EGFP expression.

Antibody responses in mice immunized with recombinant baculoviruses

Mice were vaccinated twice ( 1 and d 15) with recombinant baculoviruses and specific serum antibodies against HA were measured on d 21 using a competitive ELISA; higher ELISA readings indicate lower levels of anti-HA antibodies. As shown in Figure 5, HA-specific antibodies were produced in mice immunized with vAc-HA and vAc-HA-DUAL. No significant difference in antibody levels was observed between the two viruses, and no significant levels of HA-specific antibodies were observed in vAc-EGFP-immunized mice.

The levels of total serum IgA and IgG in immunized mice were also determined (Figure 6). vAc-HA and vAc-HA-DUAL induced 3-4 times more IgA and IgG than vAc-EGFP, and vAc-HA elicited $20 \%$ more IgA and IgG than vAc-HA-DUAL. The reason for these differences remains to be elucidated.

Production of Th1 and Th2 cytokines by splenocytes from immunized mice

The splenocytes from immunized mice were examined for the production of Th1 (IFN-Y and IL-2) and Th2 cytokines (IL-4 


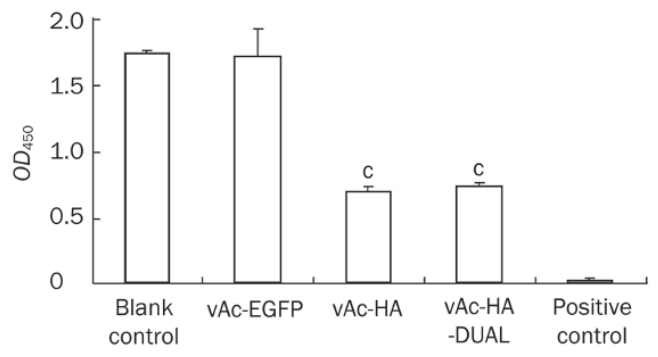

Figure 5. HA-specific antibody in serum of baculovirus-immunized mice. Sera of mice were immunized with vAc-HA-DUAL, vAc-HA, and vAc-EGFP were collected $21 \mathrm{~d}$ after the first immunization. The titers of HA specific antibody were measured by ELISA. According to the manufacturer's instruction, higher OD means negative results. Negative control and Positive control were provided by the kit. ${ }^{c} P<0.01$.

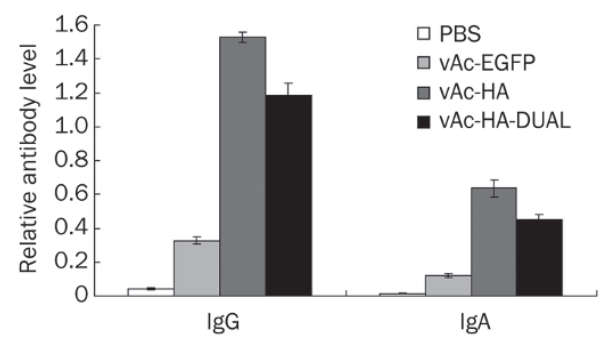

Figure 6. Total IgG and IgA antibody in immunized mice. Mice were immunized with vAc-HA-DUAL, vAc-HA, vAc-EGFP, or PBS. Sera of immunized mice from different groups were collected $21 \mathrm{~d}$ after the first immunization. The titers of total IgG and IgA antibodies were determined by ELISA.
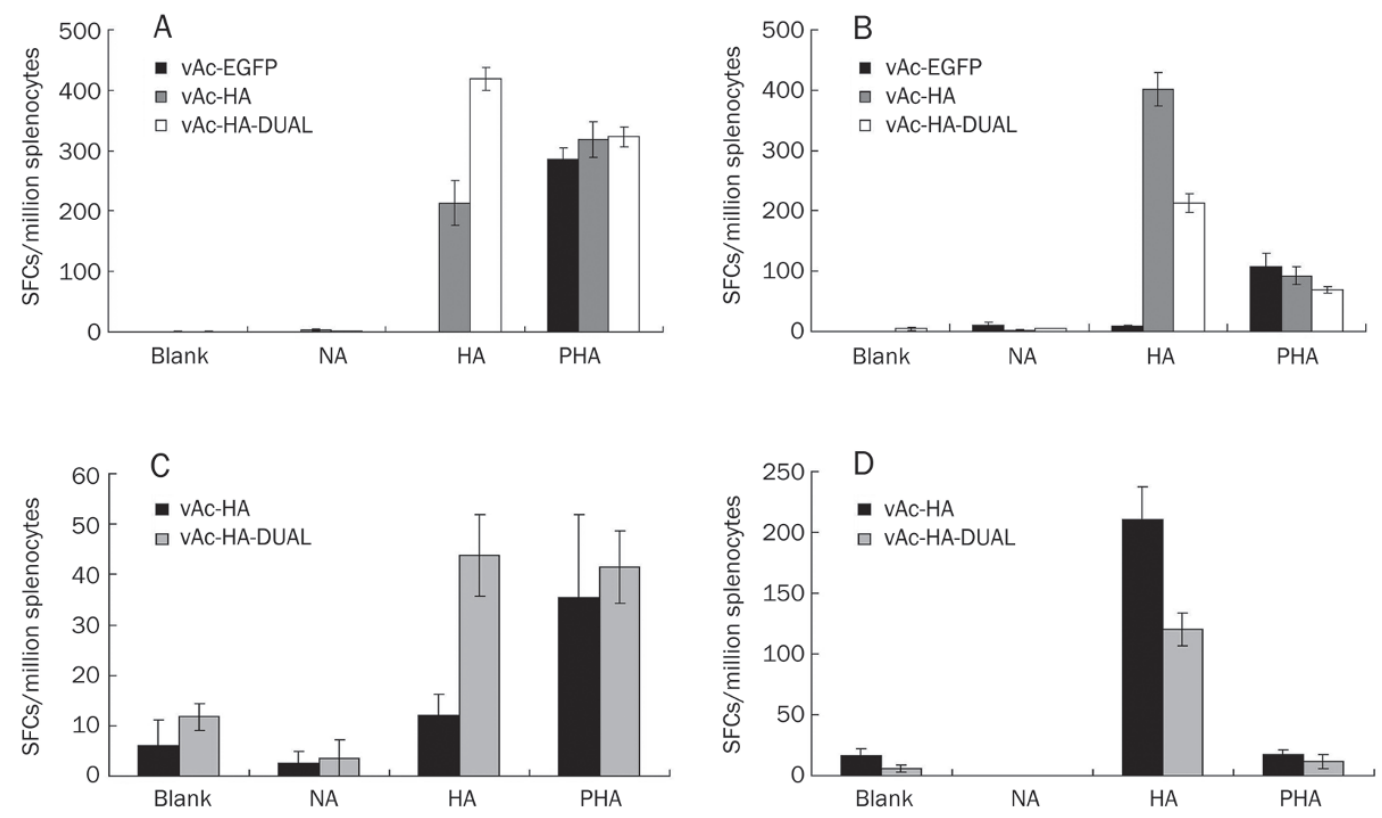

Figure 7. Number of splenocytes from baculovirus-immunized mice that produced various cytokines upon antigen stimualtion. The splenocytes of immunized mice were obtained after two immunizations and stimulated with antigen-specific peptide (HA, 14 mer), an unrelated peptide (NA, 16 mer) or plant hemaglutinin (PHA, positive control). Cytokine-producing cells were assayed with ELISPOT. Un-stimulated control (Blank) and no-cell control were also included. (A) IFN-y; (B) IL-4; (C) IL-2; (D) IL-6.

and IL-6) using ELISPOT and ELISA. As shown in Figures 7A and 7B, after stimulating with the specific antigen (a 14-mer HA peptide), the number of IFN- $\gamma$-secreting splenocytes was 20- and 40-fold higher in mice immunized with vAc-HA and vAc-HA-DUAL than in vAc-EGFP-immunized mice, respectively. Similarly, the number of IL-4-secreting splenocytes was approximately 40 - and 20-fold higher in vAc-HA and vAc-HA-DUAL mice than in vAc-EGFP mice, respectively. No differences were observed when the splenocytes were treated with an unrelated 16-mer peptide from influenza virus neuraminidase or with PHA. The results again showed that the recombinant baculoviruses elicited strong HA-specific immune responses in mice.

Furthermore, twofold more splenocytes produced IFN- $\gamma$ in mice immunized with vAc-HA-DUAL compared with mice immunized with vAc-HA (Figure 7A) whereas twofold more cells produced IL-4 in the vAc-HA group than in the vAc-HADUAL group (Figure 7B). This difference was supported by results indicating that the level of IFN- $\gamma$ was higher in splenocyte cultures from mice immunized with vAc-HA-DUAL compared with mice immunized with vAc-HA, and the level of IL-4 was higher in the vAc-HA group than in the vAcHA-DUAL group (Figures $8 \mathrm{~A}$ and $8 \mathrm{~B}$ ). Similar results were observed in separate experiments measuring the number of splenocytes producing IL- 2 and IL-6 after antigen stimulation. The number of cells producing IL-2 in the vAc-HA-DUAL group was approximately 3 -fold higher than in the vAc-HA group (Figure 7C) whereas the number of cells producing IL-6 was 2-fold higher in the vAc-HA group than in the vAc-HADUAL group (Figure 7D). These results demonstrated that 

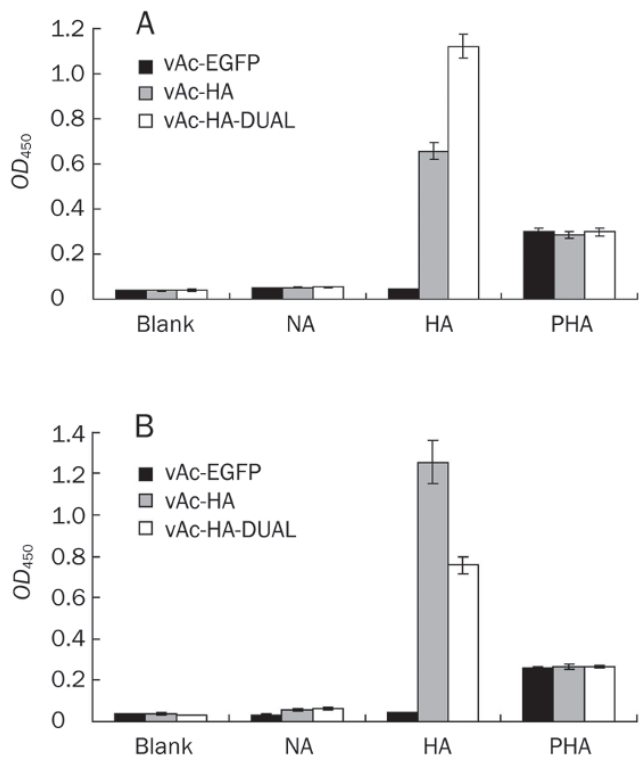

Figure 8. Cytokine production by splenocytes from baculovirus-immunized mice. Splenocytes from mice immunized with vAc-EGFP, vAc-HA, or Ac-HADUAL were isolated one week after second immunization and stimulated in vitro with peptides of HA or NA, or PHA for $72 \mathrm{~h}$. The level of IFN-y (A) and IL-4 (B) in the supernatant was analyzed by ELISA.

although both vAc-HA-DUAL and vAc-HA efficiently elicited specific immune responses, they had different biases in Th1 and Th2 responses. Specifically, vAc-HA-DUAL elicited a Th1-biased response whereas vAc-HA elicited a Th2-biased response.

\section{Discussion}

In the present study, two different recombinant baculovirus vectors were constructed and compared for their potential to induce immune responses in animals. These recombinant viruses contained the expression cassette for the H5N1 influenza virus HA gene allowing protein production in either insect cells (vAc-HA) or in both insect and mammalian cells (vAc-HA-DUAL). Both viruses mediated HA protein production in infected insect $\mathrm{Sf} 9$ cells at similar levels. HA is believed to have been transported to the surface of infected insect cells based on the observation that chicken red blood cells attached to the surface of the Sf9 cells infected by HA-expressing viruses, but not to mock-infected cells ${ }^{[15]}$ or cells infected by control virus (data not shown). This is consistent with results showing that HA proteins were present in the viral envelopes of vAc-HA and vAc-HA-DUAL and confirms previous results that HA-transportation signals were properly recognized by insect cells ${ }^{[16]}$.

The expression and surface display of HA proteins appeared to slow down the rate of viral replication in insect cells as indicated by the kinetics of budding virus production. This reduction was most likely due to the presence of HA on the virion envelope and the surface of infected cells that diluted GP64, the baculovirus major envelope protein, and thus affected viral entry and/or assembly and budding ${ }^{[17,18]}$. However, the effect seems to be limited, as the virus titer caught up at the late stage of virus infection.

On the other hand, the presence of HA on the virion surface increased the transduction efficiency of baculoviruses in mammalian cells. The percentage of cells expressing the reporter gene (egfp) was 2 times higher in A549 cells transduced by HA-displaying virus than A549 cells transduced by the control virus (vAc-EGFP). As expected, HA proteins were efficiently expressed in mammalian cells transduced by vAc-HA-DUAL but not by vAc-HA ${ }^{[19-21]}$.

In vAc-HA-DUAL, the expression of the egfp reporter and HA genes was driven by two hybrid promoters, composed either of the baculovirus $\mathrm{p} 10$ and mammalian CMV promoters, or by the baculovirus polyhedrin and CMV promoters, respectively (Figure 1). Although the transcription mediated by these hybrid promoters requires further detailed characterization, they both appeared highly effective in insect and mammalian cells ${ }^{[22,23]}$.

The recombinant baculoviruses were tested for their potential as vector vaccines to elicit immune responses against HA in mice. Because vAc-HA and vAc-HA-DUAL displayed HA proteins on the virion surface, they might act primarily as a particle vaccine. However, vAc-HA-DUAL could also work as a DNA vaccine because it could mediate HA protein expression when it entered the cells. Thus, the two virus vectors were expected to induce different immune responses.

In mice injected with vAc-HA and vAc-HA-DUAL, significant levels of HA-specific antibodies were produced. These HA-specific antibody levels were similar for both viruses, most likely indicating the same efficiency of antigen presentation for the two viruses. No HA-specific antibodies were detected in mice injected with the control virus vAc-EGFP. However, the levels of total IgG and IgA were much higher in mice injected with vAc-HA compared with mice injected with vAc-HADUAL. Very low levels of total IgG and IgA were detected in the control group. The reason for this result remains unclear.

Moreover, splenocytes from the immunized mice were cultured in vitro to test their ability to produce IFN- $\gamma$, IL-2, IL-4, and IL-6 upon stimulation with antigens consisting of an HA-specific peptide. The predominant cytokines that resulted were found to be different in mice immunized with the different baculoviruses. In mice immunized with vAc-HA, more splenocytes secreted IL-4 and IL-6 than IFN-y and IL-2, and the level of IL-4 in the culture medium was higher than the level of IFN- $\gamma$. These results suggest that vAc-HA induced a Th2-biased immune response in mice, which is consistent with the behavior of vAc-HA as a particle protein vaccine. Our results were also similar to those of previous studies using baculovirus as a vaccine vector and the surface-display approach $^{[8]}$.

On the other hand, vAc-HA-DUAL not only presented the HA protein on the virion surface but also expressed HA protein in transduced cells. Although the transduction efficiency in vivo was difficult to evaluate, the pattern of immune responses elicited by the vAc-HA-DUAL vector, as compared 
with that elicited by vAc-HA, indicated that the HA protein was likely to have been produced in vivo. Unlike vAc-HA, more splenocytes from immunized mice produced IFN- $\gamma$ and IL-2 than the Th2 cytokines IL-4 and IL-10, and the level of IFN- $\gamma$ was higher than that of IL-4 in the culture medium, suggesting a Th1-biased immune response. In addition, vAcHA-DUAL was also found to be more effective in inducing HA-specific CD4+ and CD8+ T-cell responses in vitro (data not shown).

In recent years, baculoviruses have been intensively studied as potential vaccine vectors. In addition to its high biosafety, easy handling, efficient protein production in insect cells and other advantages, baculovirus vaccine vectors have low preexisting immunity in humans and animals and the ability to elicit innate immunity. AcMNPV was found to have strong adjuvant properties. It could also activate dendritic cell (DC)mediated innate immunity through MyD88/TLR9-dependent and MyD88/TLR9-independent pathways, which might help to improve the efficacy of baculovirus vector vaccines ${ }^{[24]}$. In fact, AcMNPV was shown to induce antiviral activity in mammalian cells and confer protection against lethal encephalomyocarditis virus and influenza virus infections in mice ${ }^{[25,26]}$.

Several recent studies have shown that baculovirus transduction vectors tend to elicit a Th1-biased immune reaction against the target antigen when the antigen was only expressed in the transduced cells and was not presented on the virions. Evidence has shown that a baculovirus vector with a mammalian expression cassette producing proteins from a SARS-like coronavirus could elicit a Th1-dominant immune response in mice ${ }^{[6,13-16]}$. A similar approach has been used to develop vaccines against pseudorabies virus. High proportions of Ig2a IgG and high levels of IFN- $\gamma$ were found, indicating a Th1-type immune response. Studies using baculovirus capable of displaying circumsporozoite proteins from plasmodium on the virion surface and expressing the same protein in transduced mammalian cells demonstrated that the baculovirus induced a mixed Th1/Th2 response, resulting in strong protection against infection ${ }^{[27,28]}$. By comparing a baculovirus vector that could only display HA with a vector that could both display and express HA, we showed that different biases in Th1 and Th2 immune responses were induced. The balance between Th1 and Th2 responses mounted against an infectious agent can influence both the control of the pathogen and immunopathology. Humoral immune responses are essential for immunity against viral infections, but they may not be sufficient to clear the virus ${ }^{[6]}$. T cell responses, especially Th1-mediated cellular immunity, may also play a crucial role in long-term protection in some cases. Thus, the information provided here might be helpful for the design of effective baculovirus-based vaccines in the future.

\section{Acknowledgements}

This investigation received support from the National Natural Science Foundation of China (30770079) and the National Science and Technology Major Project for Drug Discovery (2009ZX09303).

\section{Author contribution}

Jiang ZHONG and Zhi-peng HU designed the research; Zhipeng HU, Juan YIN, Yuan-yuan ZHANG, Shu-ya JIA, and Zuo-jia CHEN performed the research; Zhi-peng $\mathrm{HU}$ and Jiang ZHONG analyzed the data; and Zhi-peng HU and Jiang ZHONG wrote the paper.

\section{References}

1 World Health Organization. Geneva: The Association; c2003-2011 [updated 2011 Aug 19; cited 2011 Oct 2]. WHO Global Influenza Programme; Available from: http://www.who.int/influenza/human_ animal_interface/H5N1_cumulative_table_archives/en/index.html

2 Chen MW, Cheng TG, Huang XY, Jan JT, Ma SH, Yu AL, et al. A onsensus-hemagglutinin-based DNA vaccine that protects mice against divergent H5N1 influenza viruses. Proc Natl Acad Sci U S A 2008; 105: 13538-43.

$3 \mathrm{Hu}$ YC. Baculovirus as a highly efficient expression vector in insectand mammalian cells. Acta Pharmacol Sin 2005; 26: 405-16.

4 Ghosh S, Parvez MK, Banerjee K, Sarin SK, Hasnain SE. Baculovirus as mammalian cell expression vector for gene therapy: an emerging strategy. Mol Ther 2002; 6: 5-11.

5 He F, Madhan S, Kwang J. Baculovirus vector as a delivery vehicle for influenza vaccines. Expert Rev Vaccines 2009; 8: 455-67.

6 Bai B, Lu X, Meng J, Hu Q, Mao P, Lu B, et al. Vaccination of mice with recombinant baculovirus expressing spike or nucleocapsid protein of SARS-like coronavirus generates humoral and cellular immune responses. Mol Immunol 2008; 45: 868-75.

7 Wang S, Fang L, Fan H, Jiang Y, Pan Y, Luo R, et al. Construction and immunogenicity of pseudotype baculovirus expressing GP5 and $\mathrm{M}$ protein of porcine reproductive and respiratory syndrome virus. Vaccine 2007; 25: 8220-7.

8 Lu L, Yu L, Kwang J. Baculovirus surface-displayed hemagglutinin of H5N1 influenza virus sustains its authentic cleavage, hemagglutination activity, and antigenicity. Biochem Biophys Res Commun 2007; 358: 404-9.

9 Kost TA, Condreay JP. Recombinant baculoviruses as expression vectors for insect and mammalian cells. Curr Opin Biotechnol 1999; 10: 428-33.

10 Kost TA, Condreay JP. Recombinant baculoviruses as mammalian cell gene delivery vectors. Trends Biotechnol 2002; 20: 173-80.

11 O'Reilly DR, Miller LK, Luckow VA. Baculovirus expression vectors. A laboratory manual, 1994. Oxford University Press, Oxford, UK.

12 Song J, Liang C, Chen X. Transduction of avian cells with recombinant baculovirus. J Virol Methods 2006; 135: 157-62.

13 Leisy DJ, Lewis TD, Leong JA, Rohrmann GF. Transduction of cultured fish cells with recombinant baculoviruses. J Gen Virol 2003; 84: 1173-8.

14 Wagle M, Jesuthasan S. Baculovirus-mediated gene expression in zebrafish. Mar Biotechnol 2003; 5: 58-63.

15 Ping W, Ge J, Li S, Zhou H, Wang K, Feng Y, et al. Baculovirusmediated gene expression in chicken primary cells. Avian Dis 2006; 50: 59-63.

16 Jin R, Lv Z, Chen Q, Quan Y, Zhang H, Li S, et al. Safety and immunogenicity of H5N1 influenza vaccine based on baculovirus surface display system of Bombyx mori. PLoS One 2008; 3: e3933.

17 Ojala K, Mottershead DG, Suokko A, Oker-Blom C. Specific binding of baculoviruses displaying gp64 fusion proteins to mammalian cells. Biochem Biophys Res Commun 2001; 284: 777-84.

18 Zhou J, Blissard GW. Display of heterologous proteins on gp64null baculovirus virions and enhanced budding mediated by a vesicular 
stomatitis virus Gstem construct. J Virol 2008; 82: 1368-77.

19 Raty JK, Airenne KJ, Marttila AT, Marjomaki V, Hytonen VP, Lehtoainen $\mathrm{P}$, et al. Enhanced gene delivery by avidin-displaying baculovirus. Mol Ther 2004; 9: 282-91.

20 Kitagawa Y, Tani H, Limn CK, Matsunaga TM, Moriishi K, Matsuura Y. Ligand directed gene targeting to mammalian cells by pseudotype baculoviruses. J Virol 2005; 79: 3639-52.

21 Ge J, Huang Y, Hu X, Zhong J. A surface modified baculovirus vector with improved gene delivery to B-lymphocytes. J Biotechnol 2007; 129: 367-72.

22 Mahonen AJ, Airenne KJ, Purola S, Peltomaa E, Kaikkonen MU, Riekkinen MS, et al. Post-transcriptional regulatory element boosts baculovirus-mediated gene expression in vertebrate cells. J Biotechnol 2007; 131: 1-8.

23 Keil GM, Klopfleisch C, Giesow K, Blohm U. Novel vectors for simultaneous high-level dual protein expression in vertebrate and insect cells by recombinant baculoviruses. J Virol Methods 2009; 160: 132-7.

24 Abe T, Hemmi H, Miyamoto H, Moriishi K, Tamura S, Takaku H, et al. Involvement of the Toll-like receptor 9 signaling pathway in the induction of innate immunity by baculovirus. J Virol 2005; 79: $2847-$ 58.

25 Gronowski AM, Hilbert DM, Sheehan KCF, Garotta G, Schreiber RD. Baculovirus stimulates antiviral effects in mammalian cells. J Virol 1999; 73: 9944-51.

26 Abe T, Takahashi H, Hamazaki H, Miyano-Kurosaki N, Matsuura Y, Takaku $\mathrm{H}$. Baculovirus induces an innate immune response and confers protection from lethal influenza virus infection in mice. J Immunol 2003; 171: 1133-9.

27 Strauss R, Huser A, Ni S, Tuve S, Kiviat N, Sow P, et al. Baculovirus based vaccination vectors allow for efficient induction of immune responses against plasmodium falciparum circumsporozoite protein. Mol Ther 2007; 15: 193-202.

28 Yoshida S, Araki H, Yokomine T. Baculovirus-based nasal drop vaccine confers complete protection against malaria by natural boosting of vaccine-induced antibodies in mice. Infect Immun 2010; 78: 595602. 